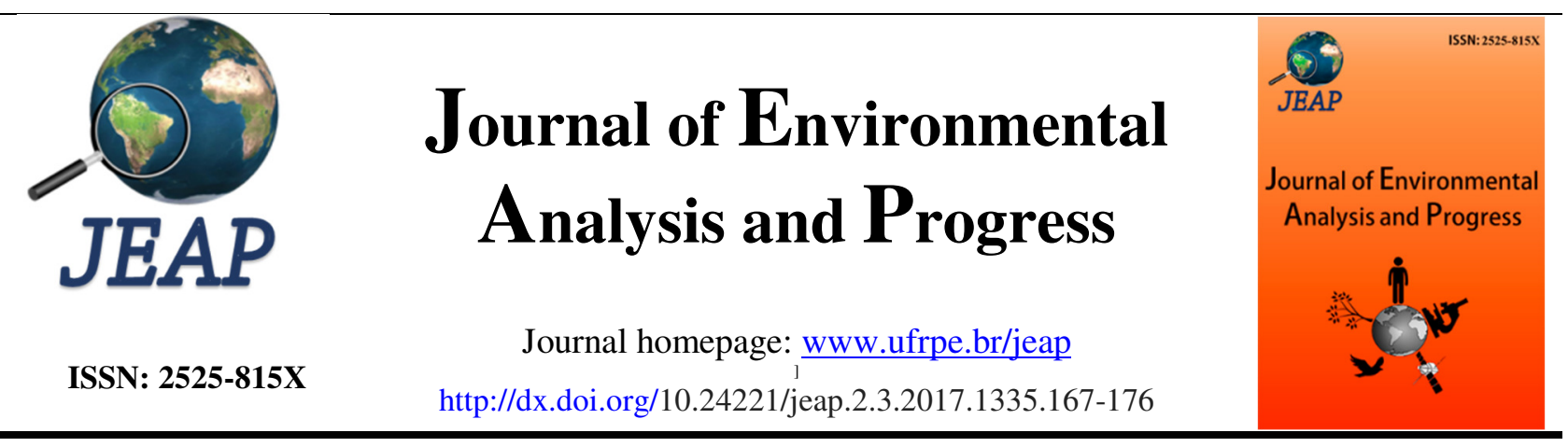

\title{
Sazonalidade na produção de serrapilheira em dois manejos no semiárido tropical
}

\section{Seasonality in production of leaf litter in two management tropical semiarid}

José Bandeira Brasil ${ }^{\mathrm{a}}$, Eunice Maia de Andrade ${ }^{\mathrm{a}}$, Deodato do Nascimento Aquino ${ }^{\mathrm{a}}$, Lécio Resende Pereira Júnior ${ }^{\mathrm{b}}$

${ }^{\text {a }}$ Universidade Federal do Ceará-UFC, Departamento de Engenharia Agrícola, Centro de Ciências Agrárias, Campus do PICI, Bloco 804, Fortaleza-CE, Brasil. CEP: 60450-760. E-mail: josebbrasil@gmail.com; eandrade.ufc @ gmail.com; deoagro@gmail.com.

${ }^{\mathrm{b}}$ UFC, Programa de Pós-Graduação em Ecologia e Recursos Naturais, Centro de Ciências, Campus do Pici, Bloco 902, Fortaleza-CE. CEP: 60450-760. E-mail: leciojunior2013@ gmail.com.

\begin{tabular}{|c|c|}
\hline A R T I C L E I N F O & A B S T R A C T \\
\hline Recebido 09 Mai 2017 & The material that is deposited throughout the year in forests has an important role in \\
\hline Aceito 22 Jun 2017 & litter formation, especially in environments under stress, as occurs in the semi-arid. \\
\hline Publicado 31 Jul 2017 & $\begin{array}{l}\text { This study aimed to identify the inference of the vegetation cover in the production } \\
\text { of leaf litter in tropical dry forest-Caatinga. The study was developed in the } \\
\text { catchment area of the Alto Jaguaribe in Iguatu-CE. It was monitored two sub-basins, } \\
\text { one with vegetation cover of caatinga in regeneration about } 38 \text { years, and another } \\
\text { one under thinning management, maintaining the plant species with stem } \\
\text { circumference } \leq 10 \mathrm{~cm} \text {. Litter samples were taken monthly (January } 2011 \text { to } \\
\text { December } 2013 \text { ). It was used } 15 \text { boxes of } 1 \mathrm{~m}^{2} \text { randomly arranged in each } \\
\text { hydrographic watershed. The average amount of leaf litter produced plots with } \\
\text { regenerating and thinned caatinga were of the order of } 4,277.2 \text { and } 2,248.0 \mathrm{~kg} \text {.ha- } \\
{ }^{1} \text {.year }{ }^{-1} \text {, respectively. Litter production presented a seasonal character, with peak } \\
\text { production immediately after the rainy season, producing respectively } 62.1 \% \text { and } \\
47.9 \% \text { of all litter in the months of May to September. It shows that the caatinga in } \\
\text { higher regeneration due to output the most individuals' ferns and most amount of } \\
\text { species. }\end{array}$ \\
\hline
\end{tabular}

Keywords: Tropical dry forest, precipitation, similarity.

\begin{abstract}
R E S U M O
O material que é depositado ao longo do ano nas florestas tem importante papel na formação da serrapilheira, especialmente em ambientes sob estresse, como ocorre no semiárido. Este estudo objetivou identificar a inferência da cobertura vegetal na produção de serrapilheira em floresta tropical seca-Caatinga. A área em estudo localiza-se na bacia hidrográfica do Alto Jaguaribe no município de Iguatu-CE. Foram monitoradas duas microbacias, uma mantida com cobertura vegetal de caatinga em regeneração há 38 anos e outra com manejo de raleamento, mantendose as espécies vegetais com circunferência do caule $\leq 10 \mathrm{~cm}$. As coletas de serrapilheira foram realizadas mensalmente (janeiro de 2011 a dezembro de 2013). Foram utilizadas 15 caixas de $1 \mathrm{~m}^{2}$ dispostas, aleatoriamente, em cada microbacia hidrográfica. A quantidade média de serrapilheira produzida nas parcelas com caatinga em regeneração e caatinga raleada foram da ordem de 4.277,2 e 2.248,0 kg.ha- ${ }^{-1}$. no $^{-1}$, respectivamente. A produção de serrapilheira apresentou um caráter sazonal, com picos de produção imediatamente posterior a quadra chuvosa, produzindo respectivamente $62,1 \%$ e $47,9 \%$ de toda a serrapilheira nos meses de
\end{abstract}


maio a setembro. Isso evidencia que a caatinga em regeneração apresentou maior produção devido a sua maior quantidade de indivíduos arbóreos adultos.

Palavras-Chave: Floresta tropical seca, precipitação, similaridade.

\section{Introdução}

No semiárido brasileiro, o recurso forrageiro de maior expressão é a vegetação de Caatinga, a qual cobre $86 \%$ da sua área, $53 \%$ da área do Nordeste e $10 \%$ do território brasileiro (IBGE, 2012). Esta vegetação desenvolve-se em região com chuvas irregulares, altas taxas de insolação e evapotranspiração (Santana \& Souto, 2011). Nesse bioma, as precipitações concentramse em duas estações bem definidas, uma seca e outra chuvosa. Para o Estado do Ceará, o qual está inserido no semiárido brasileiro, a precipitação apresenta um padrão sazonal, com um período chuvoso concentrado em três e quatro meses, podendo prolongar-se até seis meses (Guerreiro et al., 2013). Nesta condição, a floresta seca apresenta-se caducifólia logo após o término da estação chuvosa, com queda das folhas ocorrendo no início da estação seca (Amorim, Sampaio \& Araújo, 2009). O tipo de vegetação e as condições ambientais são determinantes na quantidade e qualidade do material que cai sobre o solo das florestas. O material que é depositado ao longo do ano nas florestas tem importante papel na formação da serrapilheira.

A serrapilheira é o material presente na parte superficial do piso da floresta, constituído por folhas, caules, ramos, frutos, flores e outras partes da planta, bem como restos de animais e material fecal (Nascimento, Cerqueira \& Henderson, 2015). Todo este material desempenha inúmeras funções no equilíbrio e dinâmica dos ecossistemas florestais (Costa et al., 2010; Salgado et al., 2015). É essencial na proteção do solo, principalmente na estação seca, quando as temperaturas são mais elevadas. Logo que chegam as primeiras chuvas, ele é fundamental para evitar o impacto direto das gotas da chuva no solo (Lopes et al., 2009).

Nas regiões de clima semiárido, a produção de serrapilheira é influenciada pelas estações do ano, implicando, assim, na formação da vegetação local. Autores como Lopes et al. (2009), Silva et al. (2015) e Salgado et al. (2015), estudando a produção de serrapilheira em área de caatinga, constataram que esta apresenta um pico de produção no início da estação seca, mostrando uma estreita relação com a precipitação, confirmando, deste modo, o caráter decíduo desse bioma. Visando averiguar essa relação no comportamento da serrapilheira e sua resposta em função da produção mensal em diferentes manejos, este estudo objetivou identificar a inferência da cobertura vegetal na produção de serrapilheira em floresta tropical seca - Caatinga.

\section{Material e Métodos}

$\mathrm{O}$ estudo foi conduzido em duas microbacias hidrográficas experimentais, localizadas no semiárido cearense, na bacia hidrográfica do Alto Jaguaribe, no município de Iguatu-CE. As unidades amostrais estão inseridas em uma área pertencente ao Instituto Federal de Educação, Ciência e Tecnologia do Ceará (IFCE), Campus Iguatu (Figura 1).

De acordo com a classificação climática de Köppen, o clima da região é do tipo BS (semiárido quente), e o Índice de Aridez elaborado por Thornthwaite é de 0,44 , classificando-se como semiárido. $\mathrm{O}$ valor médio de evapotranspiração potencial de 1974 a 2012 foi de 1802 mm.ano $^{-1}$, com base na metodologia Penman-Monteith/FAO (Santos et al., 2016), e a precipitação média histórica no município de Iguatu foi de $867,1 \mathrm{~mm}$ (média de 1912 a 2015) (Figura 2). A distribuição temporal das chuvas apresenta uma concentração de $85 \%$ no período de janeiro-maio, dos quais cerca de $30 \%$ são registrados no mês de março.

Uma das microbacias tem como cobertura vegetal uma caatinga em regeneração há 38 anos, sendo as especies mais frequentes aquelas pertencentes às famílias Fabaceae, com a maior riqueza florística, seguido da Euphorbiaceae e Combretaceae (Pereira Júnior, 2016). A Figura 3ab evidencia as diferenças estruturais da vegetação entre o período chuvoso e seco das parcelas com caatinga em regeneração.

A segunda microbacia foi submetida a um raleamento a cada dois anos, constituída pelas seguintes especies: Aspidosperma pyrifolium Mart., Commiphora leptophloeos (Mart.) J.B. Gillett, Mimosa caesalpiniifolia Benth., Combretum leprosum Mart. e Piptadenia stipulacea (Benth.) Ducke (Aquino, 2015). A Figura 3cd evidencia as diferenças estruturais da vegetação entre o período chuvoso e seco das parcelas com caatinga raleada. $\mathrm{O}$ raleamento consistiu em eliminar as plantas com circunferência do caule à altura do peito inferior a $10 \mathrm{~cm}$, sendo o resíduo vegetal de menor porte, galhos de menor diâmetro e ramos picotados, mantidos sobre o solo. O manejo foi realizado no mês de dezembro dos anos de 2008, 2010 e 2012. 


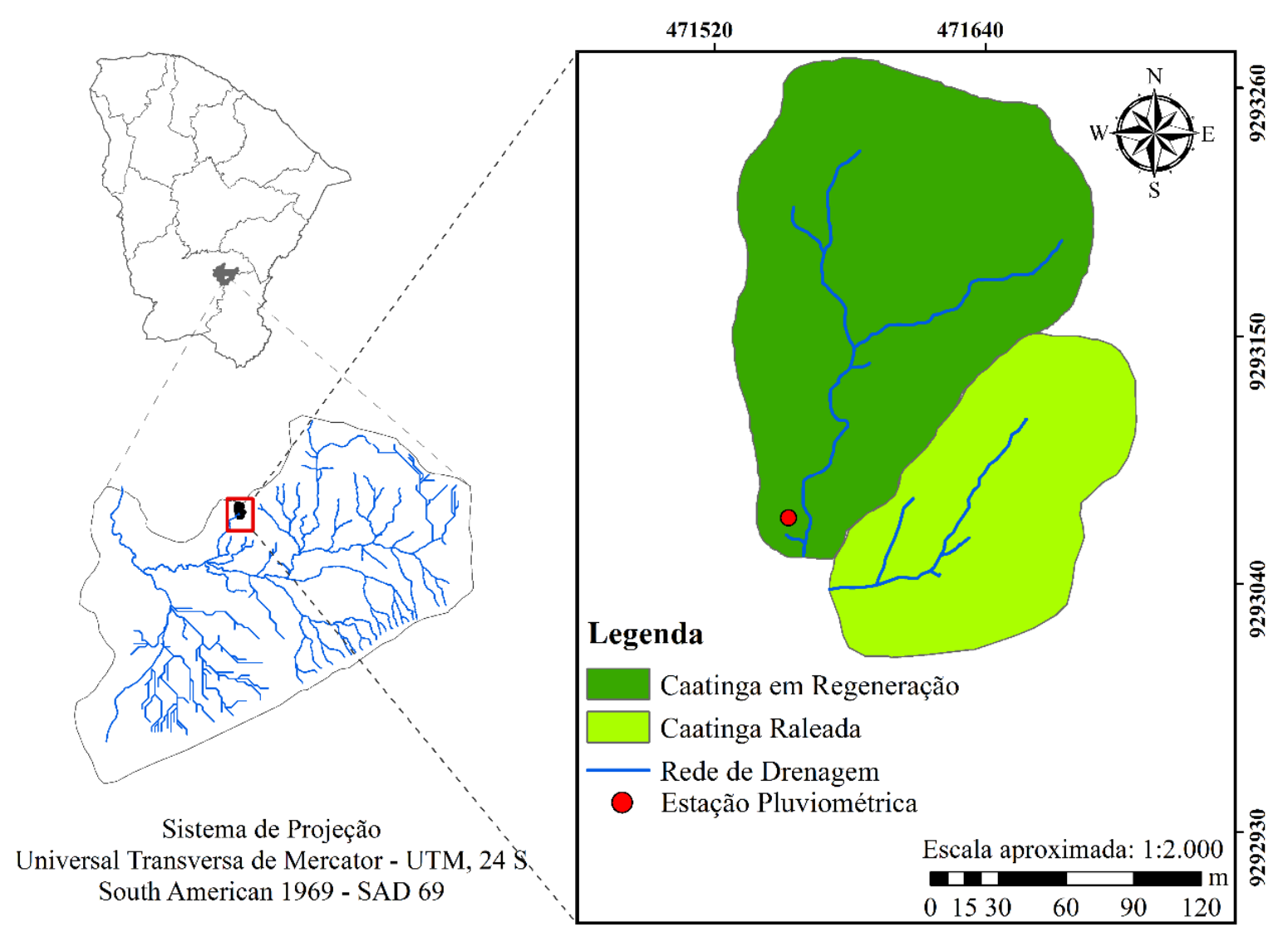

Figura 1. Localização das microbacias experimentais no município de Iguatu-CE.

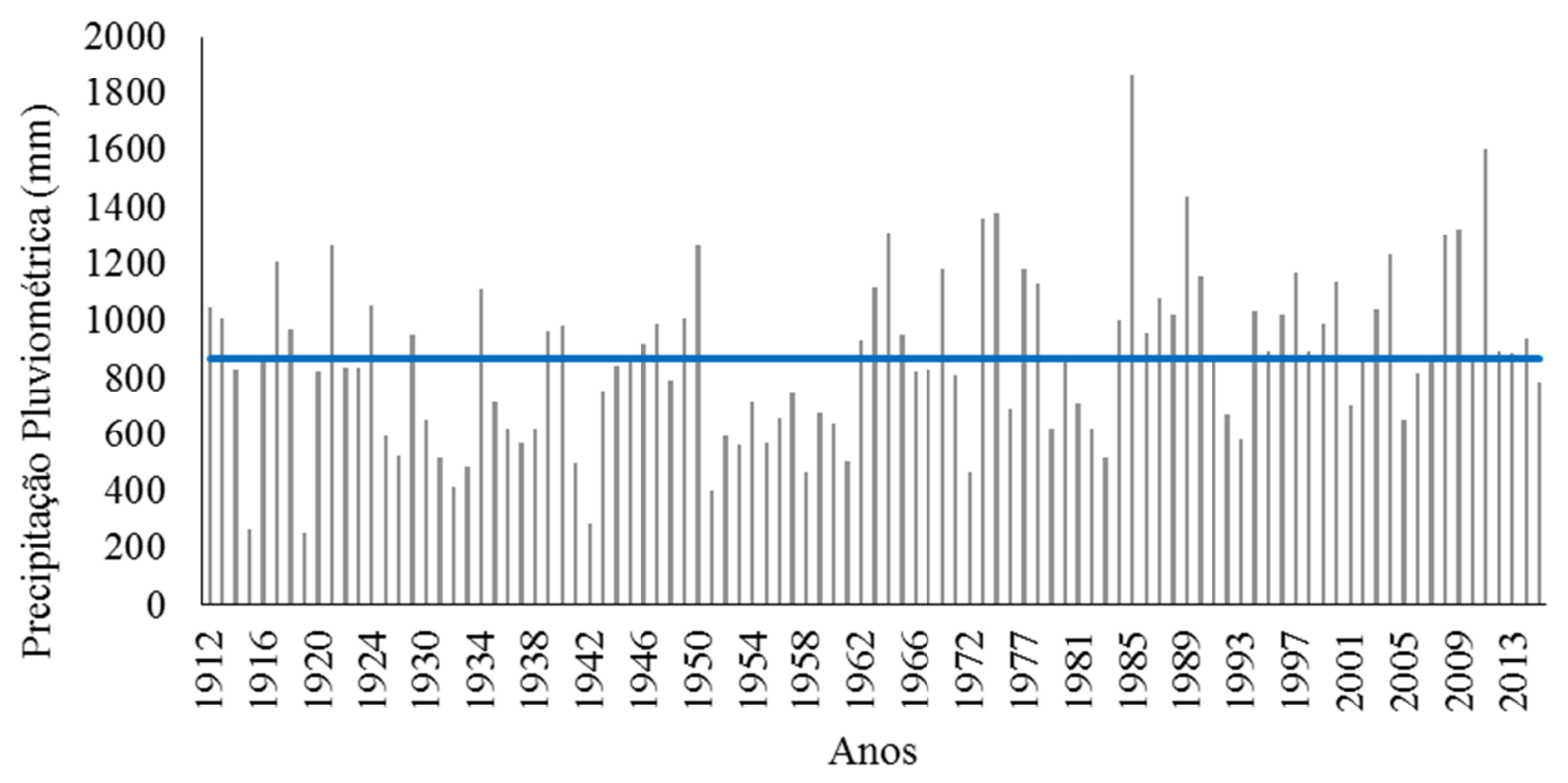

Altura pluviometrica $(\mathrm{mm}) \quad$ Média histórica

Figura 2. Série histórica anual de precipitação pluviométrica no município de Iguatu-CE.

Para a quantificação da serrapilheira presente na área de estudo foram instalados 15 coletores, distribuídos aleatoriamente, segundo o seu maior comprimento, em ambas microbacias. Cada coletor apresentou as seguintes dimensões: $1,0 \mathrm{~m}$ de largura $\mathrm{x} 1,0 \mathrm{~m}$ de comprimento $\mathrm{x} 0,25 \mathrm{~m}$ de altura (Figura 4).
O material contido nas caixas foi coletado mensalmente, de janeiro de 2011 a dezembro de 2013. Após coletado em campo, o material foi acondicionado em sacos de papel e conduzidos ao laboratório para a separação das frações: folhas, galhos, estruturas reprodutivas e miscelânea. Após a separação, procedeu-se a secagem em estufa sob 
Journal of Environmental Analysis and Progress V. 02 N. 03 (2017) 167-176

circulação forçada a $65^{\circ} \mathrm{C}$ e pesagem em balança digital. De posse das quantidades médias de serrapilheira encontradas nos coletores, estimou-se a produtividade total mensal e anual (em kg.ha $\left.{ }^{-1}\right)$.

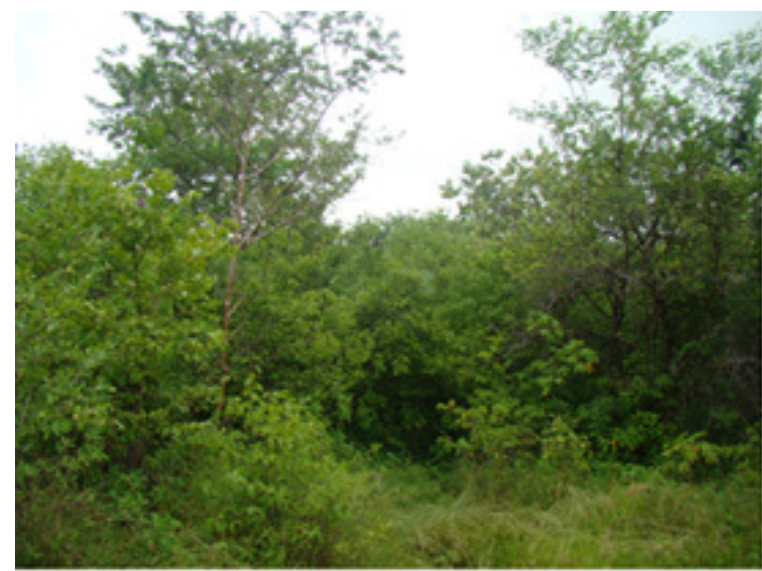

(a)

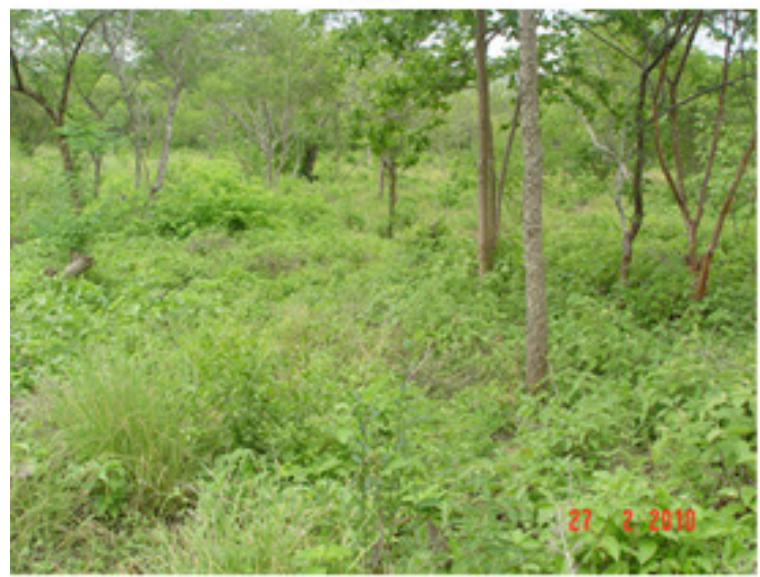

(c)

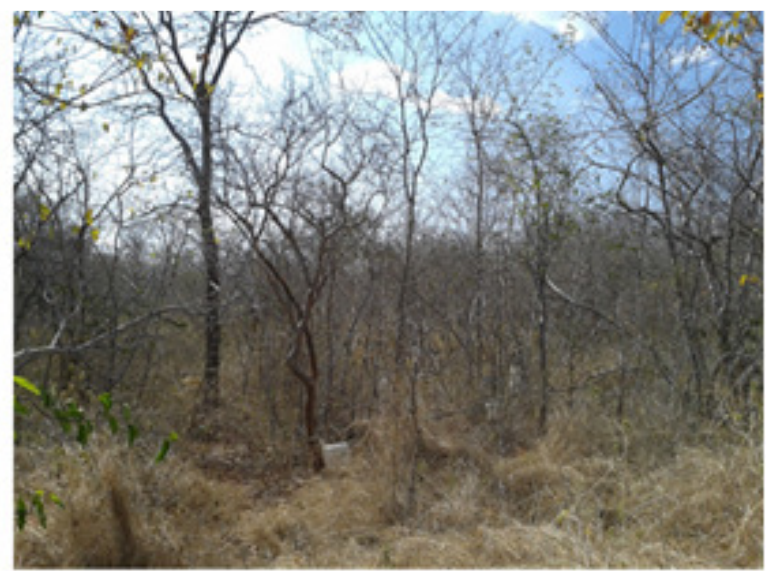

(b)

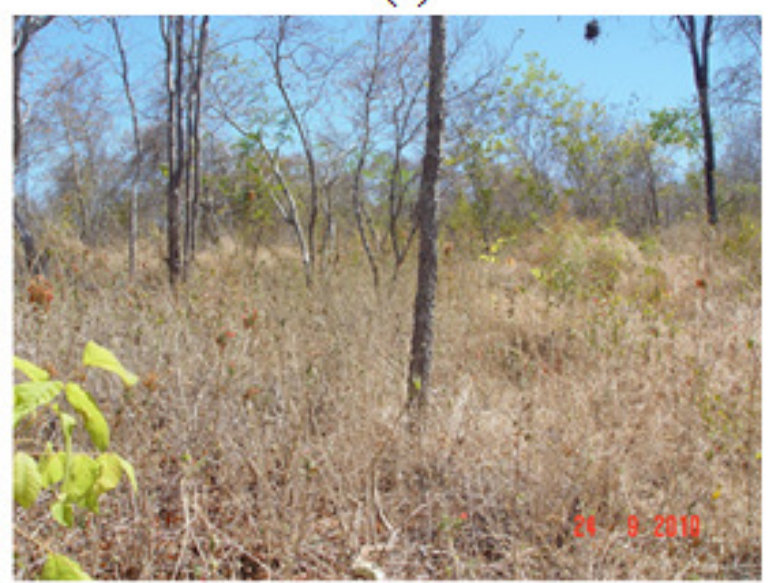

(d)

Figura 3. Imagens das áreas de caatinga, em Iguatu-CE, nas parcelas com vegetação em regeneração $(a, b)$ e raleada (c,d), no período chuvoso e seco, respectivamente.

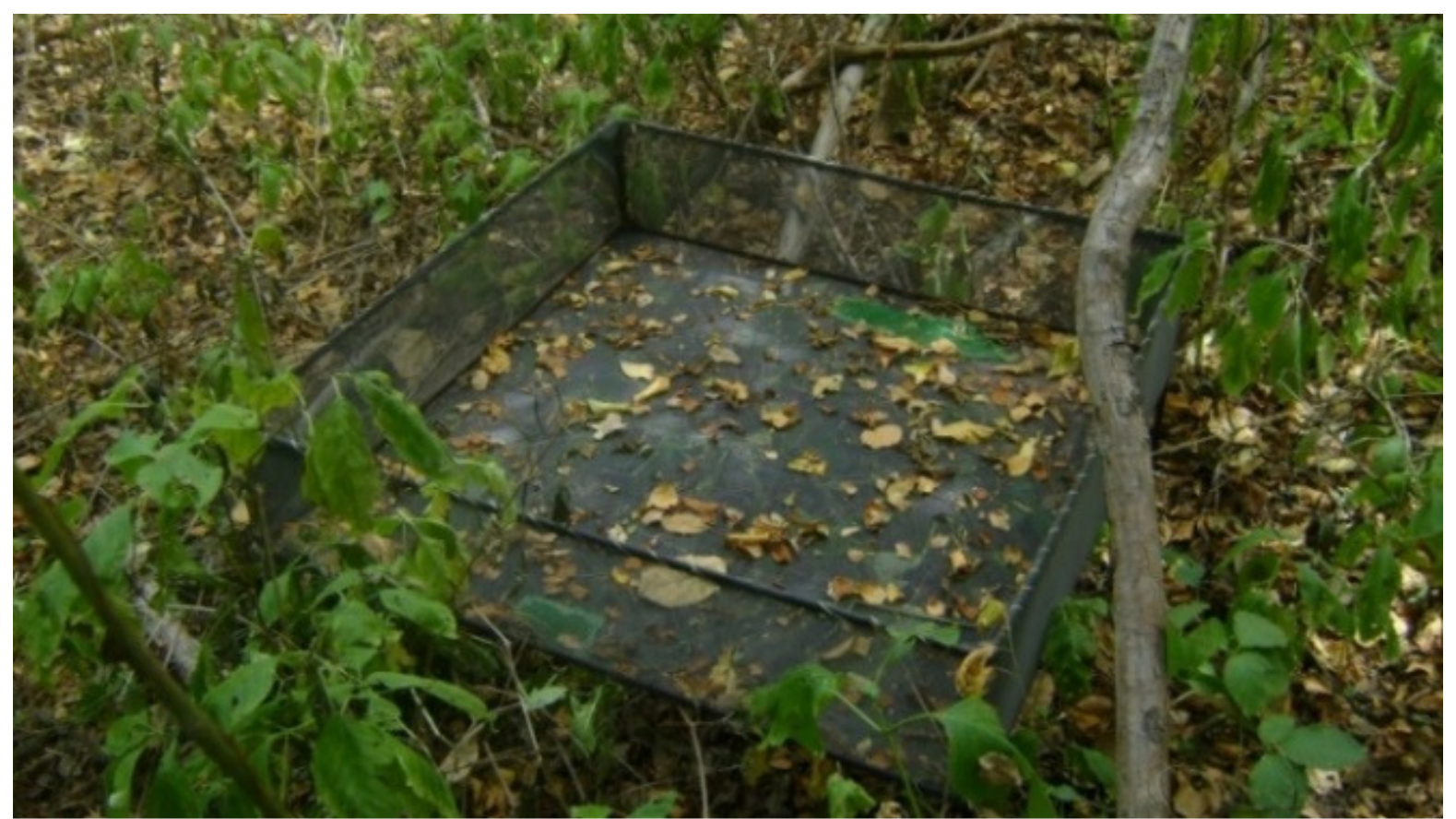

Figura 4. Caixa coletora de serrapilheira instalada na área experimental, em Iguatu-CE. 
A partir do total de serrapilheira produzido em cada mês, buscou-se entender como ocorre a distribuição das produções em função do manejo e período avaliado. Empregou-se, então, a técnica de análise multivariada, análise de agrupamento hierárquico $(\mathrm{AAH})$, pelo método aglomerativo via processamento utilizando o programa Statistical Package for the Social Sciences (SPSS), versão 16.0. Para minimizar os erros com relação às escalas e às unidades das variáveis selecionadas, foi feita a normalização dos dados (média igual a zero e desvio padrão igual a 1); a similaridade foi estimada por meio da distância Euclidiana ao quadrado. $\mathrm{O}$ algoritmo de agrupamento empregado na definição dos agregados foi o do método de Ward. Para identificar se os grupos formados pelo AAH apresentavam diferenças estatisticamente significativas, aplicou-se o teste de Wilcoxon $(\mathrm{p}<0,05)$.

\section{Resultados}

O ano de 2011 registrou a maior precipitação acumulada durante $\mathrm{o}$ período estudado, com $1.460 \mathrm{~mm}$ (Figura 5). Este acumulado representa $42,1 \%$ de toda a chuva registrada no período investigado, e no mês de abril deste mesmo ano, choveu um total $480 \mathrm{~mm}$, expressando elevada concentração dos eventos pluviométricos. Observa-se que o período chuvoso concentrou-se de janeiro a maio de cada ano. Esta característica climática se reflete, diretamente, no comportamento da vegetação local e, consequentemente, na sazonalidade da produção mensal de serrapilheira.

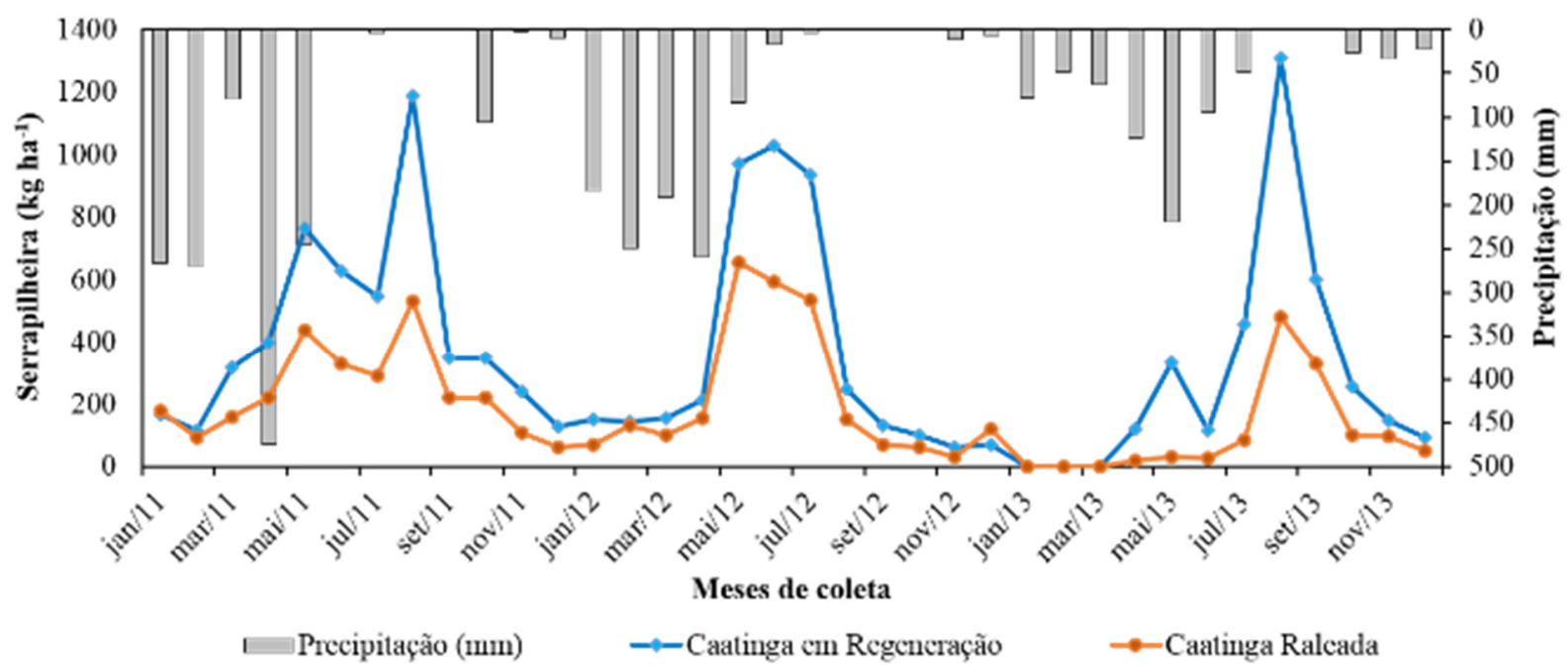

Figura 5. Representação da produção mensal de serrapilheira e a influência da sazonalidade pluviométrica em área de caatinga em regeneração e caatinga raleada, em Iguatu-CE.

A produção anual de serrapilheira para os anos de 2011, 2012 e 2013, na área com caatinga em regeneração, foi de 5.195,1, 4.201,1 e 3.435,3 kg.ha ${ }^{-1}$ ano $^{-1}$, apresentando um caráter sazonal ao longo dos meses e uma produção média de 4.277,2 kg.ha ${ }^{-1}$. Diferentes estudos conduzidos em vegetação de caatinga, comprovam essa dinamica na produção de serrapilheira (Lopes et al., 2009; Costa et al., 2015; Salgado et al., 2015). Os quantitativos médios obtidos por esses pesquisadores situam-se na faixa de $3.266,8$ a 5.365,9 kg.ha ${ }^{-1}$.

Os quantitativos de serrapilheira da área de caatinga raleada (Figura 5) apresentaram valores inferiores à da área em regeneração em praticamente todos meses monitorados. Essa maior produção na área em regeneração se deve ao fato da vegetação da caatinga mantida preservada apresentar uma maior cobertura de área foliar e, consequentemente, maior perda de folhas, principalmente nos meses em que ocorreu a senescência, proporcionando as maiores produtividades de deposição de serrapilheira nos coletores.

A produção de serrapilheira anual na área de caatinga raleada para os anos de 2011, 2012 e 2013 foi de $2.851,6,2.676,3$ e $1.216,2 \mathrm{~kg} \cdot \mathrm{ha}^{-1}$.ano 1 , respectivamente, apresentando um caráter sazonal ao longo dos meses, como verificado na área em regeneração, com uma produção média de $2.248,0 \mathrm{~kg} \cdot \mathrm{ha}^{-1}$. Essa variação espacial entre os quantitativos obtidos é devido ao comportamento da vegetação em função da pluviosidade (Salgado et al., 2015) e das espécies florestais presentes nas áreas. Portanto, fica evidenciado uma forte relação entre a precipitação e o ciclo sazonal de deposição da serrapilheira (Costa et al., 2010).

Investigando detalhadamente os dados mensais de serrapilheira e suas respostas em relação ao manejo aplicado, verificou-se que, por 
meio da análise de agrupamento, formaram-se dois grupos distintos na área de caatinga em regeneração (Figura 6), sendo definidos pela distância reescalonada de combinação entre 3,52 e 6,28 (Tabela 1). A formação de grupos distintos expressa o efeito da vegetação da caatinga em função das espécies presentes na área. $\mathrm{Na}$ estação chuvosa as espécies desenvolvem suas folhas e no periodo seco, o pico de deposição seria ocasionado pela grande quantidade de folhas liberadas pelas plantas, provocada por um estresse hídrico (Nascimento, Cerqueira \& Henderson, 2015). Além disso, destaca-se a alta variabilidade temporal dos eventos pluviométricos, bastante comuns em regiões semiáridas (Guerreiro et al., 2013).

$\mathrm{Na}$ área com caatinga raleada (Figura 7) observou-se que a maior variação ocorreu entre os coeficientes 3,096 e 6,364 (Tabela 2).

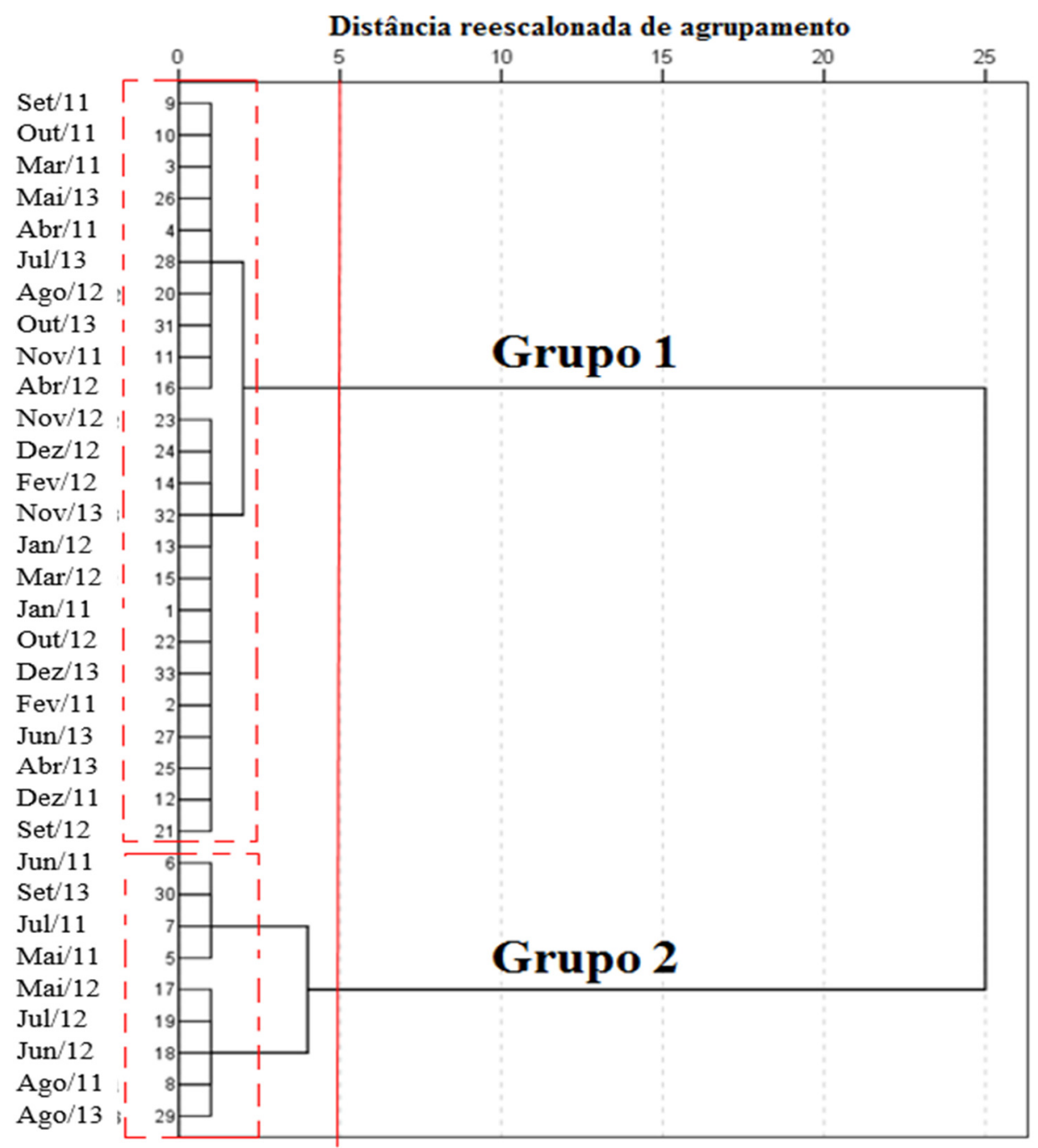

Figura 6. Dendrograma da produção mensal de serrapilheira em microbacia com caatinga em regeneração no periodo de janeiro de 2011 a dezembro de 2013, em Iguatu-CE.

Tabela 1. Variação do coeficiente de aglomeração para a análise hierárquica de agrupamentos na microbacia com caatinga em regeneração, em Iguatu-CE. $\mathrm{N}^{\circ}$. Agrup = Número de agrupamentos; Coef. = Coeficientes; D. Coef. $=$ Diferença entre coeficientes; D. Reesc. $=$ Distância reescalonada.

\begin{tabular}{cccc}
\hline $\mathbf{N}^{\circ}$. Agrup. & Coef. & D. Coef. & D. Reesc. \\
\hline 20 & 0,004 & 0,002 & 1 \\
19 & 0,006 & 0,003 & 1,002 \\
18 & 0,009 & 0,004 & 1,004 \\
17 & 0,013 & 0,005 & 1,007 \\
16 & 0,018 & 0,009 & 1,011 \\
15 & 0,027 & 0,008 & 1,017 \\
14 & 0,035 & 0,013 & 1,023
\end{tabular}


Journal of Environmental Analysis and Progress V. 02 N. 03 (2017) 167-176

\begin{tabular}{cccc}
13 & 0,048 & 0,024 & 1,033 \\
12 & 0,072 & 0,032 & 1,051 \\
11 & 0,104 & 0,032 & 1,075 \\
10 & 0,136 & 0,062 & 1,099 \\
9 & 0,198 & 0,063 & 1,146 \\
8 & 0,261 & 0,091 & 1,193 \\
7 & 0,352 & 0,176 & 1,261 \\
6 & 0,528 & 0,324 & 1,393 \\
5 & 0,852 & 0,721 & 1,636 \\
4 & 1,573 & 1,798 & 2,177 \\
3 & 3,371 & 3,681 & 3,526 \\
2 & 7,052 & 24,948 & 6,287 \\
1 & 32 & - & 25 \\
\hline
\end{tabular}

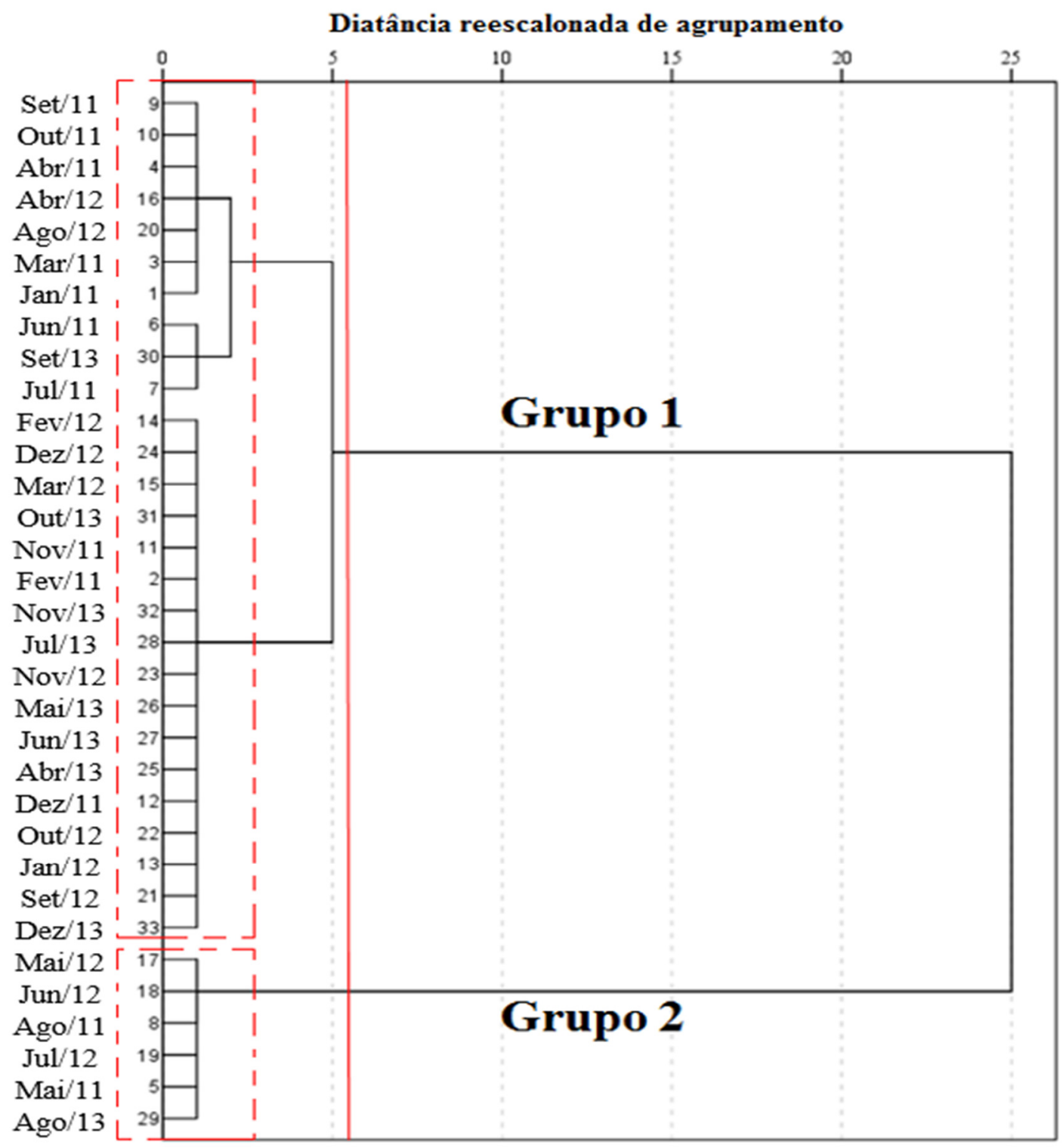

Figura 7. Dendrograma da produção mensal de serrapilheira em microbacia com caatinga raleada no período de janeiro de 2011 a dezembro de 2013, em Iguatu-CE.

Tabela 2. Variação do coeficiente de aglomeração para a análise hierárquica de agrupamentos na microbacia com caatinga raleada (CR), em Iguatu-CE.

\begin{tabular}{cccc}
\hline $\mathbf{N}^{\mathbf{0}}$. Agrup. & Coef. & D. Coef. & D. Reesc. \\
\hline 20 & 0,002 & 0,002 & 1 \\
19 & 0,004 & 0,002 & 1,002
\end{tabular}




\begin{tabular}{lccc}
18 & 0,006 & 0,001 & 1,003 \\
17 & 0,007 & 0,003 & 1,004 \\
16 & 0,01 & 0,005 & 1,006 \\
15 & 0,015 & 0,006 & 1,01 \\
14 & 0,021 & 0,012 & 1,014 \\
13 & 0,033 & 0,025 & 1,023 \\
12 & 0,058 & 0,028 & 1,042 \\
11 & 0,086 & 0,04 & 1,063 \\
10 & 0,126 & 0,057 & 1,093 \\
9 & 0,183 & 0,087 & 1,136 \\
8 & 0,27 & 0,173 & 1,201 \\
7 & 0,443 & 0,181 & 1,331 \\
6 & 0,624 & 0,427 & 1,467 \\
5 & 1,051 & 0,663 & 1,787 \\
4 & 1,714 & 1,083 & 2,284 \\
3 & 2,797 & 4,357 & 3,096 \\
2 & 7,154 & 24,846 & 6,364 \\
1 & 32 & - & 25 \\
\hline
\end{tabular}

O grupo 1 (Figura 6) foi formado pelos menores quantitativos de serrapilheira como observado na (Tabela 3). Na formação desse grupo foram contabilizadas 24 coletas mensais de serrapilheira apresentando uma máxima produção de 456,2 kg.ha ${ }^{-1}$ no mês de julho de 2013 e uma mínima de 59,8 kg.ha- ${ }^{-1}$ no mês de novembro de
2012. De acordo com a Tabela 3, a produção média mensal de serrapilheira dos meses que compõem o grupo 1 foi de 202,4 kg.ha-1. Os meses responsáveis por esses dados foram janeiro a abril e outubro a dezembro, de cada ano monitorado. Esses períodos foram caracterizados por apresentarem as menores produções de serrapilheira.

Tabela 3. Número de coletas, média, desvio padrão (DP), valores mensais, máximo e mínimo, de serrapilheira em cada grupo da microbacia caatinga em regeneração e caatinga raleada, em Iguatu-CE.

\begin{tabular}{cccc}
\hline \multirow{2}{*}{ Usos da terra } & Parâmetros & \multicolumn{2}{c}{ Serrapilheira (kg.ha-1) } \\
& & Grupo 1 & Grupo 2 \\
\hline Caatinga em & $\mathrm{N}^{\text {o }}$ de coletas & 24 & 9 \\
regeneração & Média \pm DP & $202,4 \pm 111,8 \mathrm{a}^{*}$ & $885,9 \pm 268,4 \mathrm{~b}$ \\
& Máximo & 456,2 & 1310,2 \\
& Mínimo & 59,8 & 548,6 \\
Caatinga & & & 6 \\
raleada & $\mathrm{N}^{\text {o de coletas }}$ & 27 & $539,4 \pm 78,0 \mathrm{~b}$ \\
& Média \pm DP & $129,9 \pm 89,1 \mathrm{a}$ & 655,7 \\
& Máximo & 330,3 & 438,4 \\
\hline
\end{tabular}

* Teste de Wilcoxon $(\mathrm{p}<0,05)$.

O grupo 2 (Figura 6) foi formado pelos meses que apresentaram as maiores produções de serrapilheira, mais precisamente os meses de maio a setembro. Conforme o teste de Wilcoxon $(\mathrm{p}<0,05)$, os valores médios de serrapilheira entre grupos de cada cobertura vegetal apresentaram diferença estatística. Verificou-se que o maior pico de serrapilheira ocorreu logo ao final do período chuvoso, especificamente no mês de agosto de 2013, totalizando quantitativos de $1.310,2 \mathrm{~kg} \cdot \mathrm{ha}^{-}$ ${ }^{1}$.ano ${ }^{-1}$ na área de caatinga em regeneração (Tabela $3)$.
Na Figura 7, o grupo 1 foi formado por 27 coletas mensais de serrapilheira, no qual estão inseridas as menores produções. Os quantitativos médios de serrapilheira de cada grupo apresentaram diferença estatística conforme o teste de Wilcoxon $(\mathrm{p}<0,05)$. A produção máxima registrada nesse grupo foi de $330,3 \mathrm{~kg} \cdot \mathrm{ha}^{-1} \cdot \mathrm{ano}^{-1}$ para o mês de junho de 2011 (Tabela 3). Fica evidente que a produção de serrapilheira na área raleada é inferior àquela encontrada na área de caatinga em regeneração, pois o menor quantitativo foi de 19,1 kg.ha ${ }^{-1}$. ano ${ }^{-1}$ para o mês de abril de 2013. Essa baixa produção foi em função do 
raleamento ter ocorrido no mês de dezembro de 2012 e isso influenciou diretamente, pois a vegetação no mês de abril ainda não tinha se desenvolvido. $\mathrm{O}$ grupo 2 (Figura 7) formou-se por seis coletas mensais de serrapilheira que foram responsáveis pelas maiores produções de serrapilheira. O maior pico ocorreu no mês de maio de 2012 , com uma produção de $655,7 \mathrm{~kg} \cdot \mathrm{ha}^{-1}$.ano 1 . Esse comportamento foi atípico na região que registrou as maiores precipitações nos meses de janeiro a abril, com 886,1 mm (Figura 5).

\section{Discussão}

Ao comparar os resultados do presente estudo com os de Costa et al. (2015), Lima et al. (2015) e Salgado et al. (2015), confirma-se que o total precipitado é o fator determinante da produção de serrapilheira pela vegetação.

A produção de serrapilheira no bioma caatinga apresentou um caráter sazonal, com picos de maior produção ocorrendo no início da estação seca (Lopes et al., 2009; Costa et al., 2010; Santana \& Souto, 2011; Moura et al., 2016; Silva et al., 2017) e a menor nos meses finais da mesma estação. O referido comportamento é resultado de uma diminuição da disponibilidade de água no solo para as plantas, e consequente perda das folhas e maiores taxas de deposição de serrapilheira (Moura et al., 2016; Silva et al., 2017). Alves et al. (2006) afirmam que esse comportamento é uma medida preventiva contra uma elevada perda de água por transpiração.

\section{Conclusão}

A produção de serrapilheira tem um caráter sazonal, com picos de produção imediatamente posterior à estação chuvosa, independente do manejo praticado. A maior produção na caatinga em regeneração se mostrou função da maior quantidade de indivíduos arbóreos adultos.

\section{Agradecimentos}

Os autores agradecem ao Conselho Nacional de Desenvolvimento Científico e Tecnológico-CNPq pelo apoio financeiro e à Fundação Cearense de Apoio ao Desenvolvimento Cientifico e Tecnológico-FUNCAP pelo apoio financeiro através das bolsas de produtividade e de iniciação científica.

\section{Referências}

ALVES, A. R.; SOUTO, J. S.; SOUTO, P. C.; HOLANDA, A. C. 2006. Aporte e decomposição de serrapilheira em área de caatinga, na Paraíba. Revista de Biologia e Ciências da Terra, v. 06, p. 194-203.
AMORIM, I. L.; SAMPAIO, E. V.; SÁ. B.; ARAÚJO, E. L. 2009. Fenologia de espécies lenhosas da caatinga do Seridó, RN. Revista Árvore, v. 33, p. 491-499.

AQUINO, D. N. 2015. Ciclagem de carbono e caracterização espectral em áreas de Caatinga raleada e conservada. $180 \mathrm{f}$. Tese (Doutorado em Engenharia Agrícola), Universidade Federal do Ceará, Fortaleza.

COSTA, J. T. F.; SILVA, L. S.; ALVES, A. R.; HOLANDA, A. C.; MAIA, L. M.; NUNES, A. K. A. 2015. Avaliação da serapilheira em área de mata ciliar na bacia do rio Gurguéia sul do Piauí. Revista Verde de Agroecologia e Desenvolvimento Sustentável, v. 10, p. 13-19.

COSTA, C. C. A.; DANTAS, I. M.; CAMACHO, R. G. V.; SILVA, P. C. M. 2010. Análise comparativa da produção de serapilheira em fragmentos arbóreos e arbustivos em área de caatinga na FLONA de Açu-RN. Revista Árvore, v. 34, p. $259-265$.

GUERREIRO, M. J. S.; MAIA DE ANDRADE, E.; ABREU, I.; LAJINHA, T. 2013. Long - term variation of precipitation indices in Ceará State, Northeast Brazil. International Journal of Climatology, v. 33, n. 14, p. 2929-2939.

IBGE. Mapa de Biomas e de Vegetação. 2012. Disponível em: http://www.ibge.gov.br/home/ geociencias/default_prod.shtm\#MAPAS. Acesso em: 08/05/2012.

LIMA, R. P.; FERNANDES, M. M.; FERNANDES, M. R. M.; MATRICARDI, E. A. T. 2015. Aporte e Decomposição da Serapilheira na Caatinga no Sul do Piauí. FLORAM - Revista Floresta e Ambiente, v. 22, p. 42-49.

LOPES, J. F. B.; ANDRADE, E. M. A.; LOBATO, F. A. O.; PALÁCIO, H. A. Q.; ARRAES, F. D. D. 2009. Deposição e decomposição de serapilheira em área da Caatinga. Revista Agro@ mbiente, v. 3, n. 2, p. $72-79$.

LOPES, C. G. R.; FERRAZ, E. M. N.; DE CASTRO, C. C.; DE LIMA, E. N.; DOS SANTOS, J. M. F. F.; DOS SANTOS D. M.; ARAÚJO, E. L. 2012. Forest succession and distance from preserved patches in the Brazilian semiarid region. Forest Ecology and Management, v. 271, p. 115123. 
MOURA, M. M. S.; COSTA, G. B. R.; PALÁCIO, H. A. D. Q.; ARAÚJO NETO, J. R. D.; BRASIL, J. B. 2016. Produção de serapilheira e suas frações em área da Caatinga no Semiárido Tropical. Revista Brasileira de Gestão Ambiental e Sustentabilidade, v. 3, n. 5, p. 199-208.

NASCIMENTO, L. S.; CERQUEIRA, R. M.; HENDERSON, B. L. 2015. Produção de serapilheira em um fragmento adjacente a uma cava de mineração, Ribeirão Grande, SP. Revista Brasileira de Engenharia Agrícola e Ambiental, v. 19, n. 9, p. 892-897.

PEREIRA JÚNIOR, L. R. 2016. Estrutura e estoque de carbono em vegetação de Caatinga: estudo de caso em uma área com 30 anos de regeneração. $84 \mathrm{f}$. Tese (Doutorado em Ecologia e Recursos Naturais), Universidade Federal do Ceará, Fortaleza.

SALGADO, E. V.; ANDRADE, E. M.; HEVIA, J. N.; NUNES, E. P.; RODRIGUES, M. M. A. 2015. Rainfall patterns and the contribution of litter in the caatinga dry tropical forest. Revista Ciência Agronômica, v. 46, p. 299-309.
SANTOS, J. C. N.; ANDRADE, E. M.; GUERREIRO, M. J. S.; MEDEIROS, P. H. A.; PALÁCIO, H. A. Q.; ARAÚJO NETO, J. R. 2016. Effect of dry spells and soil cracking on runoff generation in a semiarid micro watershed under land use change. Journal of Hydrology, v. 541, p. 1057-1066

SANTANA, J. A. S.; SOUTO, J. S. 2011. Produção de serapilheira na Caatinga da região semiárida do Rio Grande do Norte, Brasil. Idesia, v. 29, n. 2 p. 87-94.

SILVA, V. N.; SOUTO, L. S.; DUTRA FILHO, J. A.; SOUSA, T. M. A.; BORGES, C. H. A. 2015. Deposição de serapilheira em uma área de caatinga preservada no semiárido da Paraíba, Brasil. Revista Verde de Agroecologia e Desenvolvimento Sustentável, v. 10, p. 21-25.

SILVA, W. T. M.; LEONARDO, F. D. A. P.; SOUTO, J. S.; SOUTO, P. C.; LUCENA, J. D. S.; NETO, P. H. M. 2017. Deposição de serapilheira em áreas de Caatinga no Núcleo de Desertificação do Seridó. Agropecuária Científica no Semiárido, v. 12 , n. 4 , p. 383-390. 